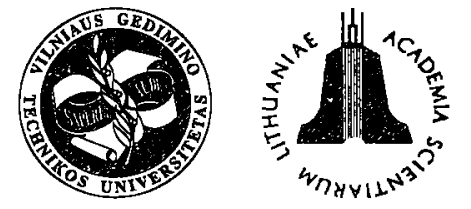

ISSN 1648-4142 TRANSPORT

http:/www.vtu.lt/english/editions

TRANSPORT - 2003, Vol XVIII, No 3, 130-135

\title{
SEARCH OF THE ROUTE IN THE NETWORK OF PUBLIC COMMUNICATION USING A LINEAR SEQUENCE METHOD
}

\author{
Artūras Keršys ${ }^{1}$, Algirdas Jurkauskas ${ }^{2}$ \\ ${ }^{1}$ Kaunas University of Technology, Kęstučio g. 27, LT-3004 Kaunas, Lithuania.E-mail: artker@centras.lt \\ ${ }^{2}$ Kaunas University of Technology, Panevėżys branch, Klaipédos g. 1, LT-5300 Panevėžys, Lithuania. \\ E-mail:algirdas.jurkauskas@mf.ktu.lt
}

Received 200301 06; accepted 20030430

\begin{abstract}
The problem of searching the route in the network of public communication is being investigated in the article. A new line sequence method working on the base of a real network model is created for route research in transport communication network and the problem solution. Having analysed a modeling peculiarity of passenger behavior choosing the route, the route evaluation and comparison of the resistance function, including the factors for the route choise are defined: traveling time, cost and comfort (number of changes). On the base of the mathematic calculation results of public transport communication a part model was determined. It offered the method of the work that can be used for the solution of the examined problem and integrated into the prognosis models of communication processes.
\end{abstract}

Keywords: public communication; the model of the network; search of the route; function of the resistance.

\section{Introduction}

The problem of searching the route in the network is one of the most important among transport service system theory tasks. The task is important itself, but its importance is enlarged by the fact that it is the composite part of the tasks connected with the optimization of flow distribution or transport network development [1]. As the shortest way algorithm usually is the main part of other tasks so its efficiency determines the effectiveness of the entire task solution [2]. It is clear, why being a big number of tasks, solving the shortest route exercises, attention to them doesn't decrease [3-5].

Two demands are raised to the route research ways. The first principal demand is not specific, a general aim is route research algorithm adaptation in the networks. This is the shortest calculation time solving the shortest route problem. The second principal demand is raised not to the route research way, but to distribution model, where route research way is integrated as a calculation component in the distribution of communication demand in the communication network [6]. This is possibly concrete and real modelling of the passenger behavior choosing the route.

There are three possibilities to upgrade the effectiveness of the route research algorithm: the search for minimal distance from one peak to another using a noniteration method, unlinear programming method adaptation and a route research algorithm, working on the base of the real network model. Implementing the first two methods in practice, the upgrade limit in the effectiveness of the research algorithm graph is reached. Though in practice, solving the specific problem, for example, the investigation of the research route in the public communication, we meet huge time expenditure, linked with the adaptation of a graphs method. So, the new route research algorithms are being created or improved the existing one, taking into account the investigated problem specific [7].

The aim of this work is to create an algorithm working on the base of the real network model. Till now there were no examples of such type of algorithm, nor their practical realization.

\section{Public Transport Communication Network Model}

Communication network allows population to travel. One of the most important factor forming the network is a transport flow, which has to be gone. A lower sufficiency limit in the communication network is estimated according to its carrying capacity and efficiency criteria. Public communication network consists of stops and lines connecting them. The network has to be coherent in order passengers from one stop could get to the other.

To foresee by what routes a journey is going on in the public communication network the connections between transport regions and stops have to be established. Transport connections are not only the consequence of the city or planned territory structures, but also the rea- 
son - transport connections and network influence each other [8]. The essence of the matter is that transport connections are the main criteria according to which communication network parameters are determined. In every region the stops belonging to the population mass centre, are attributed. So, population has one or more paths on foot to the public communication network.

Public communication network model structure formally is described so [9]:

$$
V S=(T R, S, L, P, \tau) \text {. }
$$

Distributing the explored territory into transport regions, it must be taken into account that the number of transport region stops would not exceed the determined limit, because a too large region number influences the precision of communication prognosis. Differentiation is optimal in the communication flow if every region corresponds to the stop in the entrance region and so $|T R|=|S|$. In practice it is not real because it is impossible to make the calculation of the communication flows if there are no structural data made in a micro space plane surface connected with the transport region. In spite of this it is accepted that the upper limit of stops $S \in O(T R)$ is a constant reiterative of $T R$.

Forming the line conception, as a rule it is supposed, that route length is the sequence of stops passed by and it isn't too large. So, a precondition can be made, that the number of passed by stops in a line has the upper limit

$$
\forall l \in L: l \in O(l) \text {. }
$$

Following the fact, that in the case of multiple stops passed by there is the upper limit to the passed by line number, because the formation of communication demand formation at a stop from one side and line groups, especially in the communication centers, from the other side, is limited:

$$
L \in O(S)=O(T R) \text {. }
$$

Every transport region according to ( 1 ) is connected with the stop at least by one path on foot. If there are more paths, local population can be selected according to the limited number of these alternatives, i.e. $P \in O(T R)$ is a constant reiterative of $T R$.

Usually, for most communication links in the public communication network there is no linear communication with the help of one line. So, looking for the optimal way of change, the connection has to be evaluated. It can be at every stop where at least two lines meet (so called communications points).

In the network model $V S=(T R, S, L, P, \tau)$ change links set $U$ is:

$$
U=\left\{\left(\mathrm{l}_{1}, \mathrm{l}_{2}, \mathrm{~s}\right) \in L^{2} \times \mathrm{S} \mid \mathrm{s} \in \mathrm{l}_{1}, \mathrm{~s} \in \mathrm{l}_{2} \text { and } \mathrm{l}_{1} \neq \mathrm{l}_{2}\right\} .
$$

$U$ includes all the connections of changes between separate even lines where it is necessary to change point reference. In many cases there are more change possibilities between two definite lines.

For the formal route description, firstly, lines are divided into the smallest elements - line lengths. In the network model VS line lengths set $A$ is:

$A=\left\{\left(\mathrm{s}_{i}, \mathrm{~s}_{i+1}, \mathrm{l}\right) \in S^{2} \times \mathrm{L} \mid \mathrm{l}=\left(\mathrm{s}_{1}, \ldots, \mathrm{s}_{\mathrm{n}}\right) \in \mathrm{L}, 1 \leq \mathrm{i} \leq \mathrm{n}\right\}$.

Line lengths are the connections between two lines in the route going consequently. As there can be parallel lines with different journey duration between two stops, for every length $a \in A$ reference is given for travel time differentiation. Line lengths going sequently are connected into the longer connection.

In the network model $V S$ a journey set $T$ :

$T=\left\{\left(\mathrm{s}_{i}, \mathrm{~s}_{\mathrm{j}}, 1\right) \in S^{2} \times \mathrm{L} 1=\left(s_{1}, \ldots, \mathrm{s}_{\mathrm{n}}\right) \in \mathrm{L}, 1 \leq \mathrm{i}, \mathrm{j} \leq \mathrm{n}\right\}$.

A journey is a possible connection between two stops using one of the lines. Connection $t=\left(s_{i}, \mathrm{~s}_{\mathrm{j}}, 1\right) \in T$ describes a journey from stop $s_{i}$ to the stop $s_{j}$ by the line $L$. In the route public communication network model set $K^{(V S)}$ is:

$$
K^{(V S)} \subseteq P \times T \times(U \times T)^{*} \times P .
$$

Every route begins and ends with the path on foot, including driving at least once along the line. The connection between route components has to be taken into account.

The possible route $k$ in the investigated Kaunas part public communication model from Rytai to Centras (Fig 1) is: path on foot to the stop Pastas, journey by the Ist line to the stop Kestucio, change to the 2 nd line, journey by the 2 nd line to the stop Lituanica and journey on foot to the transport region Centras.

Set $K_{\text {Rytai, Centras }}^{(V S)}$ is not finite, because there are possibilities to pass many unlimited outlines through Kestucio, Varniu and Lituanica stops.

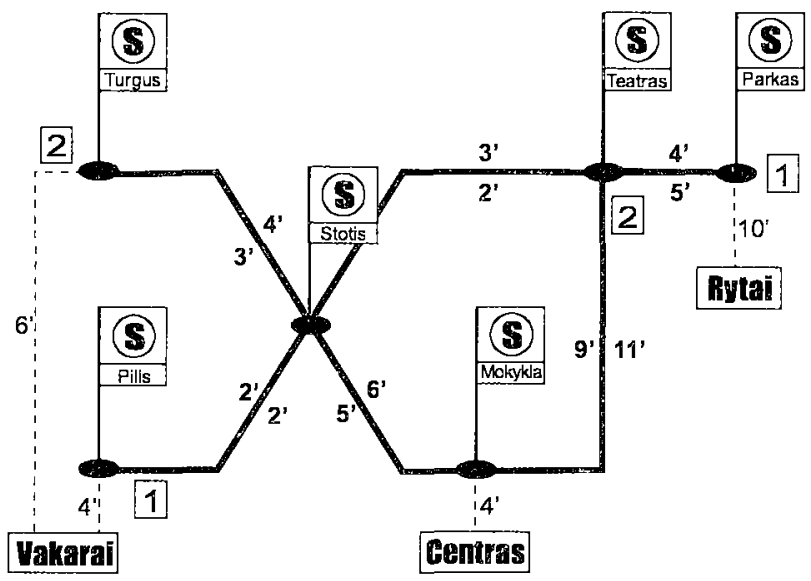

Fig 1. A public communication network model of Kaunas part 
The modelling of transport needs is based on the assumption that passengers choose the route with minimal resistance. Resistance belonging to the 1 st and the 2nd lines is calculated according to the resistance function $\omega$ defined in work [9] defined which value corresponds to leverage time.

Solving a route searching problem traditionally using the algorithm working on the base of a graph, a public communication network model will meet a need to be changed by graph: peaks instead of transport regions, stops and arches instead of paths on foot, line length and change connections. As it is seen, the size of a network model, making changes, enlarges, first of all because of the stop decomposition.

\section{Line Sequence Method}

The method is created for the searching of route selection modelling which is actual in the research of a public communication network. The algorithm works on the base of a public communication network model. The research route is from the initiate point to all other transport regions (1:N type research).

From all stops attributed to the initiate transport region going out lines are observed to their end stops and at every passed stop the covered distance is drawn (intermediate research route preservation). In this sence all the stops which are reached without changes are enrolled from the initial transport region. The second step is driving along all the lines, where at every passed stop the research route is replenished and enlarged in the line direction. So, we get stops which can be reached with a single change. The last mentioned step is done $u_{\max }$ times. In the method the accent is on the lines, so stops are researched in a line established order from the point of view of change frequency.

At every stop the drawing up is done, by what way the initial transport region can be reached. For this sake the description of the route is used.

Public communication route description set is:

$$
K A=S \times(L \times S)^{n}, \quad 0 \leq n \leq u_{\max }+1 .
$$

In this equation $u_{\max }$ is the largest allowed frequency of changes in the public communication route.

When $k=\left(s_{0}, l_{1}, s_{1}, \ldots, l_{n}, s_{n}\right) \in K A$. Where $s_{0}$ is getting in stop, $s_{n}$ - getting out stop (being $n \geq 1$ ), $s_{i}$ change stop (being $n \geq 2$ ), $l_{i}$-line used (when $1 \leq i \leq n$ ). The description of the route with $n=0$ occurs only at the beginning of a route. In algorithm they are extended successively in respect of change frequency.

Change frequency $\mu$ in public communication route description is:

$$
\mu: K A \rightarrow\left\{-1,0,1, \ldots, u_{\max }\right\} .
$$

When $k=\left(s_{0}, l_{1}, s_{1}, \ldots, l_{n}, s_{n}\right) \in K A, \mu(k)=n-1$.
In order to avoid cycle (outline) creation in the route the sequence of appearing stops must be known. To determine the stop sequence according to the route description, function $\varphi$ is taken in.

Route description stop sequence (passed stops), when $k=\left(r_{0}, l_{1}, r_{1}, \ldots, l_{m}, r_{m}\right) \in K A, \varphi: K A \rightarrow S^{+}$.

Case 1. When $m=0, \varphi(k)=\left(r_{0}\right)$.

Case 2. When $m \geq 1$ :

$$
\begin{array}{llll}
s_{A(1)+1}^{(1)}, & s_{A(1)+2}^{(1)}, & , \ldots, & s_{E(1)}^{(1)}, \\
s_{A(2)+1}^{(2)}, & s_{A(2)+2}^{(2)}, & , \ldots, & s_{E(2)}^{(2)}, \\
\ldots, & & & \\
s_{A(n)+1}^{(n)}, & s_{A(n)+2}^{(n)}, & , \ldots, & \left.s_{E(n)}^{(n)}\right) .
\end{array}
$$

The evaluation of quantitative route description made in the same way as the calculation of public communication route resistance has huge meaning for route research.

Evaluation function in route description is:

$$
\omega: K A \rightarrow I R^{+} \text {. }
$$

When $k=\left(s_{0}, l_{1}, s_{1}, \ldots, l_{n}, s_{n}\right) \in K A$ and $t r_{0} \in T R$ initial stop is:

1) when $n=0$ :

2) when $n \geq 1$ :

$$
\omega(k)=\omega\left(\left(t r_{0}, s_{0}\right)\right)
$$

$$
\begin{aligned}
\omega(k) & =\omega\left(\left(t r_{0}, s_{0}\right)\right)+g_{5} \cdot L L I+\sum_{i=1}^{n} \omega\left(\left(s_{i-1}, s_{i}, l_{i}\right)\right)+\sum_{i=1}^{n-1} \omega\left(\left(l_{i}, l_{i+1}, s_{i}\right)\right) \\
& +g_{6} \cdot \cos t+g_{7} \cdot \max \left\{\tau\left(l_{i}\right) \mid 1 \leq i \leq n\right\} .
\end{aligned}
$$

Route description resistance consists of the separate resistance of a path on foot, travel and change connection resistance, the waiting time of getting in $L L I=0,5 \cdot \min \left\{\tau\left(l_{1}\right), L L I_{\max }\right\}$, journey cost recounted into the time expression, the largest passage interval.

Public communication route list $Q_{s}$ of stop $s \in S$ is a public communication route between an initial stop $t r_{0} \in T R$ and a stop $s$ description set:

$$
\begin{gathered}
Q_{s} \subseteq\left\{k=\left(s_{0}, l_{1}, s_{1}, \ldots, l_{n}, s_{n}\right) \in K A \mid\right. \\
\left.\mid\left(t r_{0}, s_{0}\right) \in P \text { and } s_{n}=s\right\} .
\end{gathered}
$$

Public communication list of transport region $t r \in T R \backslash\left\{t r_{0}\right\}$ is a public communication route between an initial stop $t r_{0} \in T R$ and tr existing stop description set:

$$
\begin{aligned}
Q_{t r} \subseteq\left\{k=\left(s_{0}, l_{1}, s_{1}, \ldots, l_{n}, s_{n}\right) \in K A\right. \\
\left.\left(t r_{0}, s_{0}\right) \in P \text { and }\left(t r, s_{n}\right) \in P\right\} .
\end{aligned}
$$

As the number of routes $Q_{s}$ does not enlarge, significant routes are selected. Unfortunately, at the beginning of the route research, there is no absolute time limit. 
Optimal route research on the base of Dijkstra algorithm functions not on the base of a public communication network model. So a decisive criterion for selecting routes is the shortest length in the list $Q_{s}$ : alternative routes are rejected, when their resistance is too large in comparison with the urgent shortest time.

Two routes description set sampling selection $\cup_{r}$, when $d=\min \left\{\omega(k) \quad k \in M_{1} \cup M_{2}\right\}$ and

$M_{1}, M_{2} \in K A$ is:

$$
M_{1} \cup_{k} M_{2}=\left\{k \in M_{1} \cup M_{2} \mid \omega(k) \leq d+\lambda_{2}\right\} .
$$

Calculation sequence in algorithm is:

$$
Q_{s}^{(0)}, Q_{s}^{(1)}, \ldots, Q_{s}^{\left(u_{\max }\right)} \text {. }
$$

\section{Procedure:}

Given data:

Public transport communication network model $V S=(T R, S, L, P, \tau)$, initial stop $t r_{0} \in T R$.

To be searched:

All public communication routes $k \in L_{t r_{0}, t r}^{(V S)}$ in all transport regions $\operatorname{tr} \in T R \backslash\left\{t_{0}\right\}$; routes are drawn into the list $Q_{t r}$.

Formation:

$\forall s \in S:$

IF $\left(\mathrm{tr}_{0}, \operatorname{tr}\right) \in \mathrm{P}$ THEN $\mathrm{Q}_{\mathrm{s}}^{(0)}:\{(\mathrm{s})\}$,

ELSE $Q_{S}^{(0)}:=\{\}$ END -recording of all attached stops.

Basic part:

FOR $\mathrm{u}:=0$ TO $\mathrm{u}_{\max }$ DO

$$
\forall \mathrm{l}=\left(\mathrm{s}_{1}, \mathrm{~s}_{2}, \ldots, \mathrm{s}_{\mathrm{n}}\right) \in \mathrm{L} \text { : }
$$

$\mathrm{R}:=\{\}$;

FOR i: $=1$ TO $\mathrm{n}-1$ PO

$\left.\mathrm{R}:=\mathrm{R} \cup \cup_{\mathrm{r}}\left\{\mathrm{k} \in \mathrm{Q}_{\mathrm{s}_{\mathrm{i}}}^{\mathrm{u}}\right) \mid \mu(\mathrm{k})=\mathrm{u}-1\right\}$

$\forall \mathrm{k} \in \mathrm{R}$ :

IF $\mathrm{s}_{\mathrm{i}+1} \in \varphi(\mathrm{k})$ THEN R: $=\mathrm{R} \backslash\{\mathrm{k}\}$

END;

ELSE $\mathrm{Q}_{\mathrm{s}_{i+1}}^{(\mathrm{u}+\mathrm{Y})}:=\mathrm{Q}_{\mathrm{s}_{\mathrm{i}+1}}^{(\mathrm{u})} \cup_{\mathrm{r}} \mathrm{k} \cdot\left(1, \mathrm{~s}_{\mathrm{i}+1}\right)$ END

END;

END;

END.

Found routes are determined to every end point: $\forall \operatorname{tr} \in \mathrm{TR} \backslash\left\{\mathrm{tr}_{0}\right\}$. The attached stop research route is recorded: $\mathrm{Q}_{\mathrm{tr}}:=\{\} ; \forall(\mathrm{tr}, \mathrm{s}) \in \mathrm{P}: \mathrm{Q}_{\mathrm{tr}}:=\mathrm{Q}_{\mathrm{tr}} \cup_{\mathrm{r}} \mathrm{Q}_{\mathrm{s}}^{\left(\mathrm{u}_{\max }\right)}$.

Correctness proof:

Such solution set is determined, for every stop $s \in S$ that is, all noncycle routes are found from the initial stop $t r_{0}$ to the stop s with time limit $d_{t r_{0}, s}+\lambda_{2}$ and the largest change frequency $u_{\max }$ :

$$
\begin{aligned}
& X_{s}=\left\{k \in K A \varphi(\mathrm{k})=\left(s_{1}, s_{2}, \ldots, s_{n}\right)\right. \\
& \text { with }\left(\operatorname{tr}_{0}, s_{1}\right) \in P, s_{n}=s, \forall 1 \leq x \leq n: s_{x} \neq s_{y}, \\
& \text { and } \left.\omega(\mathrm{k}) \leq d_{t r_{0}, s}+\lambda_{2} \text { and } \mu(\mathrm{k}) \leq u_{\max }\right\} .
\end{aligned}
$$

Notice, that after executing the algorithm calculation to the route list, such equality is valid:

$$
\forall s \in S: Q_{s}^{\left(u_{\max }\right)}=X_{s} .
$$

With the help of induction through outer cycle (outline) calculating step u can prove, that $Q_{s}^{(u)}$ is all the routes from $X_{s}$ with the largest change amount $u$.

Proposition $\beta(u)$ :

$$
\begin{aligned}
& \forall s \in S \text {, when } d=\min \left\{\omega(k) Q_{s}^{(u)}\right\}: \\
& Q_{s}^{(u)}=\left\{k \in K A \quad \varphi(\mathrm{k})=\left(s_{1}, s_{2}, \ldots, s_{n}\right)\right. \\
& \text { with }\left(\operatorname{tr}_{0}, s_{1}\right) \in P, s_{n}=s, \forall 1 \leq x \leq n: s_{x} \neq s_{y}, \\
& \text { and } \left.\omega(\mathrm{k}) \leq d+\lambda_{2} \text { and } \mu(\mathrm{k}) \leq u\right\} .
\end{aligned}
$$

The beginning of induction $(u=0)$.

Forming algorithms reached:

$$
\forall s \in S, \forall s \in S: \mathrm{Q}_{\mathrm{s}}^{(0)}:=\{(s)\},\left(\operatorname{tr}_{0}, \mathrm{~s}\right) \in P \text {. }
$$

The description of routes $Q_{s}^{(0)}$ is the direct connection between initial stop and stop $s$ and are noncycled. Their length does hot overstep the upper limit $d+\lambda_{2}$, because a shorter connection does not exit. So, the proposition is fulfilled.

Induction condition (IC):

When $0 \leq u \leq u_{\max }-1$, valid $\beta(u)$.

Induction proposition: $\beta(u+1)$ instead of $u$.

$\forall s \in S$, when $d=\min \left\{\omega(k) Q_{s}^{(u+1)}\right\}$ :

$Q_{s}^{(u+1)}=\left\{k \in K A \quad \varphi(\mathrm{k})=\left(s_{1}, s_{2}, \ldots, s_{n}\right)\right.$

with $\left(\operatorname{tr}_{0}, s_{1}\right) \in P, s_{n}=s, \forall 1 \leq x \leq n: s_{x} \neq s_{y}$,

and $\omega(\mathrm{k}) \leq d+\lambda_{2}$ and $\left.\mu(\mathrm{k}) \leq u+1\right\}$.

Induction step.

Any stop is analysed $s \in S$. In the cycle revision $u$ all the lines are going along. Lines which do not go through $s$ stop are not important, because they do not change $Q_{s}^{(u+1)}$. So analysed $\forall l=\left(s_{1}, \mathrm{~s}_{2}, \ldots, \mathrm{s}_{\mathrm{n}}\right) \in L$ with $s \in l$, when $1 \leq x \leq n, s_{x}=s$.

When $x=1, l$ is not important, because $Q_{s}^{(u+1)}$ does not change.

When $x>1$, in case of inner cycle revision $i=x-1$ selected connection is being done:

$$
R=\bigcup_{r=1}^{i}\left\{k \in Q_{s_{r}}^{(u)} \mu(k)=u\right\} .
$$

Let suppose, that $M=\left\{k \cdot\left(l, s_{i+1}\right) k \in R\right\}$ is given. When $s_{i+1}=s_{x}=s$, then $M=\{k \cdot(l, s) k \in R\}$.

In case of connection, every route description is replenished by one journey, that is the additional change is got. As change frequency $u$ for every route $k \in R$ : 


$$
\begin{aligned}
& \forall k \in M: \mu(k)=u+1, s_{i+1}=s \notin \varphi(k): \\
& Q_{s_{i+1}}^{(u+1)}=Q_{s}^{(u+1)}=Q_{s}^{(u)} \cup M .
\end{aligned}
$$

According to induction condition $-\forall k \in Q_{s}^{(u)}$ :

$\forall k \in Q_{s}^{(u)}:$ and $k$-noncycled.

So, for every route $k \in Q_{S}^{(u+1)}$ valid:

$\mu(k) \leq u+1$;

$k$ is noncycled $(s \notin \varphi(k))$;

when $d=\min \left\{\omega(k) Q_{s+1}^{(u)}\right\}, \omega(k) \leq d+\lambda_{2}$ is

valid.

Proposition is proved in such a way.

Estimation of expenses:

In order to evaluate the costs it is important to fulfill the condition:

$$
\exists k \in I N:\left(\mathrm{R} \leq k \text { and } \forall \mathrm{s} \in \mathrm{S}: \mathrm{Q}_{\mathrm{s}} \leq k\right) .
$$

Calculating procedure parameters:

- Formation in $|S|$ steps : $\in O(N)$;

- Include research route to $R$ in $k$ steps: at every stop;

- Cycle revision $s_{i+1} \in \varphi(k)$ in $\log _{2}(|S|)$ steps;

- Copy of research route in $|S|$ steps;

- Inventory a new route into the list in $\log _{2}(k)$ steps;

- Certified cycle number $u_{\max }|L| \cdot$ constk $: \in O(N \cdot k)$;

- $\quad$ End in $T R \cdot$ const steps: $\in O(N)$.

Total expenses of the method:

$$
\begin{aligned}
& |S|+u_{\max }|L| \text { constk }+k\left(\log _{2}(|S|)+|S|+\log _{2}(k)\right)+|T R| \cdot \text { const } E \\
& \quad \in\left(N^{2} \cdot k\right) .
\end{aligned}
$$

Total expenses for the route research $N: N$ (method is repeated $N$ times): $\in O\left(N^{3} \cdot k\right)$.

Total expenses are the size of the same line, as in the case of research in depth [9]. Line observation quality is such, that no longer public communication network change by graph arises and the number of stops in the line is less than the number of peaks and arches in the equivalent graph of a network model.

\section{Occupied memory size:}

Dividing algorithm in terms the size of route list $Q_{s}$ is decisive, because for all the stops $s \in S$ at the same time determination of $k$ routes to $|S|$ stops is being done. The size of memory is $O\left(|S|^{2} \cdot k\right)=O\left(N^{2} \cdot k\right)$.

\section{Programming Realization of the Line Sequence Al- gorithm}

Programming language Delphi for operational system Windows was used for the programming line sequence algorithm realization of the route. Data system reflects the interrelation connections between network elements. Network elements (stops and other) as objects are distributed into six classes (Fig 2).

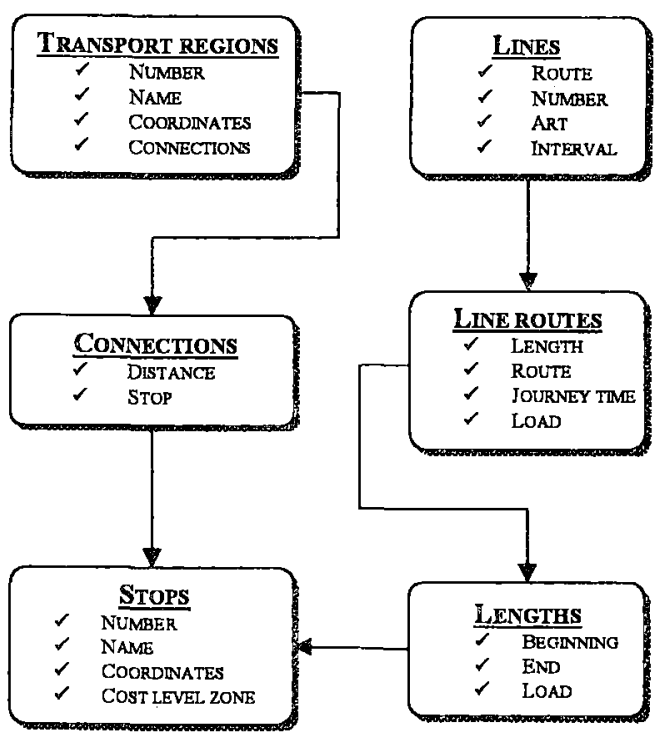

Fig 2. Public transport communication data structure

In transport regions there are connections which indicate stops. Stops are connected by lengths, lengths by line routes. The indicator reflects reference to the object of other class. Connections are saved as transport region connections with a peak. Connections in the reverse direction are not examined, because they are redundant. Also, double connection between a transport region and a peak would have a negative influence on the size of memory.

A line is saved as a line route sequence, but not as a stops sequence. So it is easier to operate with travel time, distance of carriages and load data. Line routes are situated in lengths that are the connections between two stops. Only stops which have at least one length route are examined. In case of lengths the direction and reverse direction are ruled commonly because in all the lengths the transportation is done in both directions.

In comparison with a public transport service network model, graph data structure creation is a simpler task because there are only two object classes: peaks and arches. From the other side, graphs are more bulky because stops and transport regions have to be divided into more arches.

The end transport region is reached through the list sequence which is coordinated with the transport region sequence according to its number (Fig 3).

The route data according to its size is the longest and the most important program set. It has the descriptions of all found routes. A pair list for every transport region is foreseen. As their number can be very large, forming data records memory saving is the main task.

The duration of route $k$ involvement to the research sequence $R$ depends on the research route number (ex- 


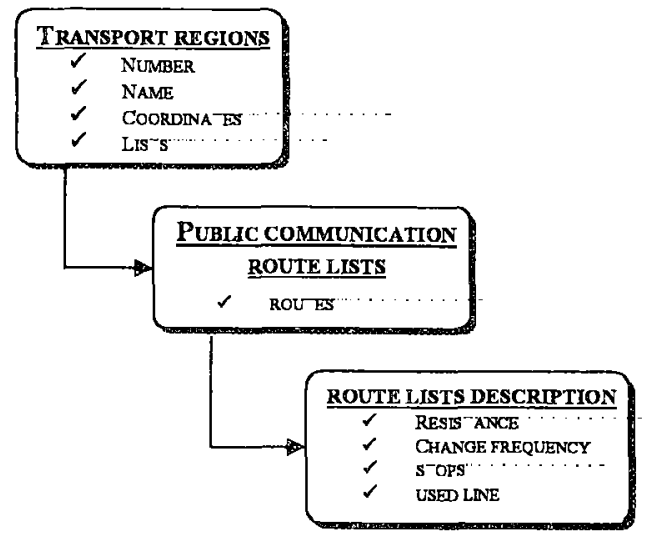

Fig 3. The structure of public transport communication route lists

treme case $-N$ ). Binary insert needs $\log _{2}(N)$ step. By "Basket sort" method (divide and gather) grading expenses are being reduced per step; for every meaning $i \in\{0,1, \ldots, n\}$ set is foreseen $M_{i}$. Set vector $\left(M_{i}\right)_{i \in\{0,1, \ldots, n\}}$ is called divisor. The shortest actual research route is the way from not empty set $\forall 0 \leq j<i$ : $M_{j}=\{\}$. Resistance, as a rule, is not-integral number $\left(\gamma(k) \in I R^{+}\right)$, very large divisors are given, which correspond to rounded route length.

Verifying cycles to find the stop in the set $\log (N)$ step is needed. Verifying can be done by one step, when additional binary $|S|$ list is being created. Process duration of the route $k$, insertion to the list $Q_{S}$ is reduced by one step using a "basket sort" method. Every stop has its divisor.

In the work the efficiency of the investigated method as well as the evaluation in practice, experimental calculations of the research public trans-port communication network model for route are done.

Public transport service network is a real Kaunas city transport communication route fragment with 15 stops, 6 transport regions, 22 lines, 34 transport ties and changing frequency 0,2 [9]. The calculation data were received from the Transport Problems Institute special group who was responsible for passenger flow research in Kaunas.

Calculation lasts for $5,2 \mathrm{sec}$. The created line set method successfully competes with a traditional task solution method. The method calculation is based on a public transport communication network model, so there is no necessity of changing it by graph.

\section{Conclusions}

1. A new line sequence method working on the base of a real network model is created for route research in transport communication network and for the problem solution.
2. Having analysed the modelling peculiarity of passenger behavior choosing the route, for the route evaluation and the comparison of the resistance function, including the factors for the route choise are defined: traveling time, cost and comfort (number of changes).

3. Kaunas city public transport communication part model was determined. On the base of mathematic calculation results it offers the method that can be used for the solution of the examined problem and integrated into the prognosis models of transport service processes.

4. There is no need to replace a public communication network model by graph: the stop number in comparison with a peak number in graph is less 21 times, line length number in comparison with arches number is less 14 times.

5. The created line sequence method can be successfully used for the optimal route research in spite of the necessity to save the research route at every stop and the resistance recalculation of getting in and changing for every line.

\section{References}

1. Baublys, A. Introduction to the theory of transport system (Transporto sistemos teorijos ivadas). Vilnius: Technika, 1997. 298 p. (in Lithuanian).

2. Magnadi, T. L.; Wong, R. T. Network design and transportation planning models and algorithms. Transportation Science, 1984, Vol 18, No 1, p. 3-55.

3. Nguyen Sang. An algorithm for the traffic assignment problem. Transportation Science, 1974, Vol 8, No 3, p. 163192.

4. Dievulis, G. Method of finding the shortest path in the transport network. Transport Engineering (Transportas), Vol XV, No 1, Vilnius: Technika, 2000, p. 3-11 (in Lithuanian).

5. Goldman, A. J.; Nemhauser, G. J. A transport improvement problem transformable to a best-path problem. Transportation Science, Vol 1, No 1, 1967, p. 295-307.

6. Dinic, E. Economical algorithms of finding of the shortest paths in the network. Works of VNIISI, 1978, No 4, p. 3644 (in Russian).

7. Theune, D. Strong and effective methods to solution of problems of path (Robuste und effiziente Methoden zur Lösung von Wegproblemen). B. Teubner Verlag, 1995 (in German).

8. Wermuth, M. Representations of model for prediction. Planning of the city traffic - bases, methods, the purposes (Modellvorstellungen zur Prognose. Stadverkehrsplanung - Grundlagen, Methoden, Ziele). Springer Verlag Heidelberg, 1994, p. 221-274 (in German).

9. Keršys, A.; Jurkauskas, A. Search for the optimal way in the network of public communication. Transport, Vol XVII, No 2, Vilnius: Technika, 2002, p. 60-65. 